\title{
Resolvent Estimates and Scattering States for Dissipative Systems
}

By

\author{
Mitsuteru KADOWAKI*
}

\section{$\S 1 . \quad$ Introduction}

Mochizuki [17] has studied the scattering problem for wave equations with dissipative terms (see also Nakazawa [19]). In this paper we shall study the similar problem for wave equations with dissipative terms in stratified media.

To explain our wave equations, we state acoustic operators in stratified media.

Let $n \geqq 1$ and $(x, y) \in \mathbf{R}^{n} \times \mathbf{R}$. We set

$$
c_{0}(y)= \begin{cases}c_{+} & (y \geqq h) \\ c_{h} & (0<y<h) \\ c_{-} & (y \leqq 0),\end{cases}
$$

for some positive constants $h$ and $c_{+}, c_{-}, c_{h}$.

Acoustic operators are

$$
L_{0}=-c_{0}(y)^{2} \triangle
$$

where

$$
\triangle=\sum_{j=1}^{n} \frac{\partial^{2}}{\partial x_{j}^{2}}+\frac{\partial^{2}}{\partial y^{2}} .
$$

Considering the case $c_{h}<\min \left(c_{+}, c_{-}\right)$we find the guided waves (cf. Wilcox [28] or Weder [27]).

$L_{0}$ is a positive self-adjoint operator in $\mathcal{G}=L^{2}\left(\mathbf{R}^{n+1} ; c_{0}(y)^{-2} d x d y\right) . D\left(L_{0}\right)$ is given by $H^{2}\left(\mathbf{R}^{n+1}\right), H^{s}\left(\mathbf{R}^{n+1}\right)$ being Sobolev space of order $s$ over $\mathbf{R}^{n+1}$.

Communicated by T. Kawai. Received February 5, 2001. Revised June 12, 2001.

2000 Mathematics Subject Classification(s): Primary 35P25; Secondary 47A40.

* Tokyo Metoropolitan College of Aeronautical Engineering, Arakawa-ku, Tokyo 116-0003, Japan.

e-mail: kadowaki@kouku-k.ac.jp 
We deal with the following dissipative wave equations:

$$
\partial_{t}^{2} u(x, y, t)+b(x, y) \partial_{t} u(x, y, t)+L_{0} u(x, y, t)=0
$$

and

$$
\partial_{t}^{2} u(x, y, t)+\left\langle\partial_{t} u, \varphi\right\rangle_{\mathcal{G}} \varphi(x, y)+L_{0} u(x, y, t)=0,
$$

where $(x, y, t) \in \mathbf{R}^{n} \times \mathbf{R} \times[0, \infty)$ and $\langle\cdot, \cdot\rangle_{\mathcal{G}}$ is the inner-product of $\mathcal{G}$.

We assume that $b(x, y)$ and $\varphi(x, y)$ are measurable functions which satisfy

$$
0 \leqq b(x, y) \leqq C\left(1+|x|^{2}+y^{2}\right)^{-\frac{\theta}{2}}
$$

and

$$
\varphi(x, y) \in L^{2}\left(\mathbf{R}^{n+1} ;\left(1+|x|^{2}+y^{2}\right)^{\frac{\theta}{2}} d x d y\right)
$$

for some $\theta>1$ and $C>0$.

We shall show the existence of the scattering states for (1.1) and (1.2) which are considered as the perturbed systems of

$$
\partial_{t}^{2} u(x, y, t)+L_{0} u(x, y, t)=0, \quad(x, y, t) \in \mathbf{R}^{n} \times \mathbf{R} \times \mathbf{R}
$$

(see Theorem 3.1). [17] has already dealt with the case $c_{h}=c_{+}=c_{-}=1$ and $n \geqq 2$ of (1.1). His proof has been based on Kato's smooth perturbation theory [14] and some resolvent estimates for $L_{0}$ as in Proposition 1.0 below.

Proposition 1.0. Assume that $c_{h} \leqq c_{+}=c_{-}$. Then there exist positive constants $C$ and $\eta$ such that

$$
\sup _{0<|\operatorname{Im} \zeta|<\eta}|\zeta|\left\|X_{\frac{\theta}{2}}\left(L_{0}-\zeta^{2}\right)^{-1} X_{\frac{\theta}{2}}\right\|_{L^{2}\left(\mathbf{R}^{n+1}\right) \rightarrow L^{2}\left(\mathbf{R}^{n+1}\right)} \leqq C,
$$

where $\zeta \in C, X_{\gamma}=\left(1+|x|^{2}+y^{2}\right)^{-(\gamma / 2)}$ and $\|\cdot\|_{L^{2}\left(\mathbf{R}^{n+1}\right) \rightarrow L^{2}\left(\mathbf{R}^{n+1}\right)}$ is the norm of the bounded operator in $L^{2}\left(\mathbf{R}^{n+1}\right)$.

Proof. For the case $c_{+}=c_{h}=c_{-}$, see Kuroda [16] Theorem 4.4.1, Corollary 4.4.3 and those proof (see also [17] Proposition 2.1 and that proof). For the case $c_{h}<c_{+}=c_{-}$, see Kadowaki [12] Theorems 1.1, 1.3 and these proof.

Theorem 1.1 of $[12]$ is stated low energy part $(|\zeta| \rightarrow 0)$ for the case $c_{h}<$ $\min \left(c_{+}, c_{-}\right)$. Ben-Artzi, Dermenjian and Guillot [2] has also considered low energy estimate for the case $\theta \geqq 1$. In [12] the condition $c_{+}=c_{-}$is needed to get the estimate of the high energy part $(|\zeta| \rightarrow \infty)$. It seems that this condition is a technical one. However another case, $c_{h}<\min \left(c_{+}, c_{-}\right)$and $c_{+} \neq c_{-}$, have 
not been dealt yet. In generally it is not easy to get the estimate of (1.4) type. For instance, considering $c_{h}<\min \left(c_{+}, c_{-}\right)$we have to analyze thresholds (cf. [28], [27] and [12]).

In this paper, to show the existence of scattering states we use the following resolvent estimates of $L_{0}$.

Proposition 1.1. Assume that the positiveness of $c_{+}, c_{h}, c_{-}$only. Then for any $\beta>\alpha>0$, there exist positive constants $C_{\alpha, \beta}$ and $\eta$ such that

$$
\sup _{\alpha \leqq|\operatorname{Re} \zeta| \leqq \beta, 0<|\operatorname{Im} \zeta|<\eta}\left\|X_{\frac{\theta}{2}}\left(L_{0}-\zeta^{2}\right)^{-1} X_{\frac{\theta}{2}}\right\|_{L^{2}\left(\mathbf{R}^{n+1}\right) \rightarrow L^{2}\left(\mathbf{R}^{n+1}\right)} \leqq C_{\alpha, \beta} .
$$

Proof. See DeBièvre and Pravica [5] Theorem 1.1, [6] Theorem 2.1 (a) and these proof (see also Zhang [29] Theorem 2.2).

In Proposition 1.1 we do not restrict ourselves to special cases, for instance $c_{h}<\min \left(c_{+}, c_{-}\right)$or $c_{h}=c_{+}=c_{-}$.

As results of the resolvent estimates of $L_{0}$, there are [27], Ben-Artzi, Dermenjian and Guillot [1], Boutet de Monvel-Berthier and Manda [3] too.

We shall give an abstract framework which is used the idea of [17]. Applying our framework to (1.1) and (1.2) we show the existence of the scattering states for those equations (see Section 3). Then (1.5) will be used.

Below we state our framework.

Let $\mathcal{H}$ be a separable Hilbert space with inner product $\langle\cdot, \cdot\rangle_{\mathcal{H}}$. This norm is denoted by $\|\cdot\|_{\mathcal{H}}$. Let $A_{0}$ and $B$ be self-adjoint operators in $\mathcal{H}$. We suppose that $B$ is non-negative, $D\left(A_{0}\right) \subset D(B)$ and the following assumptions.

(A1) $\sigma\left(A_{0}\right)=\sigma_{a c}\left(A_{0}\right)=\mathbf{R}$ or $[0, \infty)$.

(A2) $B$ is $A_{0}$-compact.

(A3) $\sqrt{B}\left(A_{0}-\zeta\right)^{-1} \sqrt{B}$ can be extended to a bounded operator $Q(\zeta)$ which satisfies that for any $\beta>\alpha>0$, there exist positive constants $C_{\alpha, \beta}$ and $\eta$ such that

$$
\sup _{\alpha \leqq|\operatorname{Re} \zeta| \leqq \beta, 0<|\operatorname{Im} \zeta|<\eta}\|Q(\zeta)\| \leqq C_{\alpha, \beta},
$$

where $\zeta \in C$ and $\|\cdot\|$ is the norm of the bounded operator in $\mathcal{H}$.

According to [14], [15] or Reed and Simon [24], (1.6) implies that

$$
\sup _{\alpha \leqq|\operatorname{Re} \zeta| \leqq \beta, 0<|\operatorname{Im} \zeta|<\eta}|\operatorname{Im} \zeta||| \sqrt{B}\left(A_{0}-\zeta\right)^{-1} \|^{2} \leqq C_{\alpha, \beta}
$$

and (1.7) means that $B$ is $A_{0}$-local smooth (see also (2.1) of Section 2). 
We set $A=A_{0}-i B, D(A)=D\left(A_{0}\right)$. Then Reed and Simon [23] (see Theorem X-50) implies that $A$ generates a contraction semi-group, $\{V(t)\}_{t \geq 0}(V(t)=$ $\left.e^{-i t A}\right)$. The unitary group generated by $A_{0}$ is denoted by $\left\{U_{0}(t)\right\}_{t \in \mathbf{R}}\left(U_{0}(t)=\right.$ $\left.e^{-i t A_{0}}\right)$.

Remark 1.2. If $(\sqrt{B})^{-1}$ is densely defined, it follows from (A3) and [24] (see Theorem XIII-20) that $A_{0}$ is absolutely continuous. Then we may suppose

$$
\sigma\left(A_{0}\right)=\mathbf{R} \quad \text { or } \quad[0, \infty)
$$

instead of (A1).

Abstract result is

Theorem 1. Let $A_{0}$ and $B$ be self-adjoint operators in $\mathcal{H}$. Assume that $B$ is non-negative, $D\left(A_{0}\right) \subset D(B)$ and $(\mathrm{A} 1) \sim(\mathrm{A} 3)$. Then

(1) A has no real eigenvalues.

(2) The wave operator

$$
W=s-\lim _{t \rightarrow \infty} U_{0}(-t) V(t)
$$

exists. Moreover $W$ is not zero as an operator in $\mathcal{H}$.

We shall use (1.5) to get (1.6) or (1.7) (see Section 3).

Theorem 1(2) implies that there exist scattering states of $d V(t) g / d t=$ $-i A V(t) g, g \in D(A)$ as follows:

Corollary 2. Let $A_{0}$ and $B$ be self-adjoint operators in $\mathcal{H}$. Assume that $B$ is non-negative, $D\left(A_{0}\right) \subset D(B)$ and $(\mathrm{A} 1) \sim(\mathrm{A} 3)$. Then there exist non-trivial initial data $f \in \mathcal{H}$ and $f_{+} \in \mathcal{H}$ such that for any $k=0,1,2, \cdots$, and $\zeta_{0} \in \boldsymbol{C}$ satisfying $\Re \zeta_{0}>0$

$$
\lim _{t \rightarrow \infty}\left\|V(t)\left(A-\zeta_{0}\right)^{-k} f-U_{0}(t)\left(A_{0}-\zeta_{0}\right)^{-k} f_{+}\right\|_{\mathcal{H}}=0 .
$$

The proof of Theorem 1 and Corollary 2 will be given in Section 2 .

Abstractly, Mochizuki's result [17] can be explained as follows. Let $A_{0}$ and $B$ be self-adjoint operators in $\mathcal{H}$. If we suppose that $B$ is non-negative, bounded and there exist positive constants $C$ and $\eta$ such that

$$
\sup _{0<|\operatorname{Im} \zeta|<\eta}\left\|\sqrt{B}\left(A_{0}-\zeta\right)^{-1} \sqrt{B}\right\| \leqq C
$$

instead of (A1), (A2) and (A3), we can prove the same conclusion as in Theorem 1 (see Lemma 2.1 of Section 2). 
(1.8) implies that

$$
\sup _{0<|\operatorname{Im} \zeta|<\eta}|\operatorname{Im} \zeta|\left\|\sqrt{B}\left(A_{0}-\zeta\right)^{-1}\right\|^{2} \leqq C
$$

(cf. [14], [15] or [24]). (1.9) means that $B$ is $A_{0}$-smooth.

We give some remarks concerning our results and the framework as in [17]. The proof of (1.6) or (1.7) is easier than that of (1.8) or (1.9). [17] does not argue the conclusion as in Corollary 2 because he uses no compactness like (A2). From these points of view, our abstract result is a extension of framework of [17].

Previous framework [13] using Enss method (cf. Enss [8], Simon [25] and Perry [20]) has not been applied to (1.1) and (1.2) with $c_{h}<\min \left(c_{+}, c_{-}\right)$. Since generalized eigenfunctions of acoustic wave propagation in stratified media with $c_{h}<\min \left(c_{+}, c_{-}\right)$have thresholds (cf. [28] or [27]), the key estimates of [13] has not been obtained in the neighborhood of each threshold (cf. Kadowaki [11]). Present framework can be applied the wider class of unperturbed systems than the ones in previous framework [13]. But the class of perturbation in [13] (for instance, elastic wave with dissipative boundary conditions in $\mathbf{R}_{+}^{3}$ ) is wider than the class in this paper.

We shall also consider Schrödinger equations with absorption as applications of present framework (see Section 3). In this paper we give only two applications. However present framework can be applied to other wave equations with dissipative terms, for instance acoustic wave in two unbounded media (cf. Eidus [7] and Kadowaki [10]) and elastic wave in a half space (DermenjianGuillot [4]) or stratified (two-layered) media (cf. Shimizu [22]). Scattering problem in exterior domain as in Mochizuki-Nakazawa [18] can also be considered.

Considering scattering problem for dissipative systems we have to note decay states (see Section 2 (2.10)). It seems that there is little literature which study dissipative systems with energy scattering and decay states, for instance, Georgiev [9] and Stefanov-Georgiev [26]. They have dealt with Maxwell equations with dissipative boundary conditions in exterior domains. In Section 4 we shall given an example of (1.2) which has energy decay (see (4.9) and (4.10)) and scattering states. Energy decay states will be constructed by using eigenfunctions associated with complex eigenvalues of the reduced wave equation from (1.2) (cf. Perry [20] P. 47 and P. 92). The existence of scattering states will be due to Theorem 1 (2). 


\section{§2. Proof of Theorem 1 and Corollary 2}

For the sake of simplicity, we shall restrict ourselves to the case $\sigma\left(A_{0}\right)=$ $\sigma_{a c}\left(A_{0}\right)=\mathbf{R}$ only.

Let $E(\lambda)$ be the spectral family of $A_{0}$. Then we have

$$
A_{0}=\int_{-\infty}^{\infty} \lambda d E(\lambda) .
$$

For $\beta>\alpha>0$, we denote $E((-\beta,-\alpha) \cup(\alpha, \beta))$ by $E_{\alpha, \beta}\left(A_{0}\right)$.

(A3) implies that $\sqrt{B} E_{\alpha, \beta}\left(A_{0}\right)$ is $A_{0}$-smooth, i.e. for any $g \in \mathcal{H}$

$$
\int_{-\infty}^{\infty}\left\|\sqrt{B} U_{0}(t) E_{\alpha, \beta}\left(A_{0}\right) g\right\|_{\mathcal{H}}^{2} d t \leqq \tilde{C}_{\alpha, \beta}\|g\|_{\mathcal{H}}^{2}
$$

(cf. Kato [14], Kato and Yajima [15] or Reed and Simon [24]), where $\tilde{C}_{\alpha, \beta}$ is a positive constant which depends on $\alpha$ and $\beta$ only. Moreover we note the following identity of $V(t) f, f \in D(A)$ :

$$
\|V(t) f\|_{\mathcal{H}}^{2}+2 \int_{0}^{t}\|\sqrt{B} V(\tau) f\|_{\mathcal{H}}^{2} d \tau=\|f\|_{\mathcal{H}}^{2},
$$

Using (2.1) and (2.2) we prove the following lemma.

Lemma 2.1. Let $\beta>\alpha>0$. Then for any $f \in D(A)$ one has

$$
\lim _{t, s \rightarrow \infty}\left\|E_{\alpha, \beta}\left(A_{0}\right)\left(U_{0}(-t) V(t)-U_{0}(-s) V(s)\right) f\right\|_{\mathcal{H}}=0 .
$$

Proof. The proof is same way as in Mochizuki [17] Section 3. So we give a brief sketch of the proof only.

For any $f \in D(A)$ and $g \in \mathcal{H}$ we have

$$
\begin{aligned}
& \left\langle E_{\alpha, \beta}\left(A_{0}\right)\left(U_{0}(-t) V(t)-U_{0}(-s) V(s)\right) f, g\right\rangle_{\mathcal{H}} \\
& =-\int_{s}^{t}\left\langle\sqrt{B} V(\tau) f, \sqrt{B} U_{0}(\tau) E_{\alpha, \beta}\left(A_{0}\right) g\right\rangle_{\mathcal{H}} d \tau .
\end{aligned}
$$

Thus Schwarz inequality and (2.1) imply

$$
\begin{aligned}
\| E_{\alpha, \beta}\left(A_{0}\right)\left(U_{0}(-t) V(t)\right. & \left.-U_{0}(-s) V(s)\right) f \|_{\mathcal{H}} \\
& \leqq\left(\tilde{C}_{\alpha, \beta}\right)^{\frac{1}{2}} \times\left(\int_{s}^{t}\|\sqrt{B} V(\tau) f\|_{\mathcal{H}}^{2} d \tau\right)^{\frac{1}{2}} .
\end{aligned}
$$

It follows from (2.2) that

$$
\int_{0}^{\infty}\|\sqrt{B} V(\tau) f\|_{\mathcal{H}}^{2} d \tau \leqq\|f\|_{\mathcal{H}}^{2} .
$$


By (2.3) and (2.4) the proof is complete.

By Lemma 2.1 and (A1) we have the following lemma.

Lemma 2.2. One has

$$
w-\lim _{t \rightarrow \infty} V(t)=0 .
$$

Proof. Since $D(A)$ is dense in $\mathcal{H}$, it is sufficient to show that for any $f \in D(A)$ and $g \in \mathcal{H}$

$$
\lim _{t \rightarrow \infty}\langle V(t) f, g\rangle_{\mathcal{H}}=0
$$

Let $M>1$. Then we have

$$
\begin{aligned}
&\left|\left\langle\left(U_{0}(-t) V(t)-U_{0}(-s) V(s)\right) f, g\right\rangle_{\mathcal{H}}\right| \\
& \leqq\|f\|_{\mathcal{H}} \times\left\|\left(I_{d}-E_{1 / M, M}\left(A_{0}\right)\right) g\right\|_{\mathcal{H}} \\
& \quad+\left\|E_{1 / M, M}\left(A_{0}\right)\left(U_{0}(-t) V(t)-U_{0}(-s) V(s)\right) f\right\|_{\mathcal{H}} \times\|g\|_{\mathcal{H}}
\end{aligned}
$$

Therefore noting that Lemma 2.1 and for any $\varepsilon>0$, there exists $M>1$ such that

$$
\left\|\left(I d-E_{1 / M, M}\left(A_{0}\right)\right) g\right\|_{\mathcal{H}}<\varepsilon,
$$

we have by $(2.5)$ that there exists $\tilde{f}_{+} \in \mathcal{H}$ such that

$$
\lim _{t \rightarrow \infty}\left\langle U_{0}(-t) V(t) f, g\right\rangle_{\mathcal{H}}=\left\langle\tilde{f}_{+}, g\right\rangle_{\mathcal{H}}
$$

Taking $s \rightarrow \infty$ in (2.5) and putting $g=U_{0}(t) h$ for any $h \in \mathcal{H}$, we have

$$
\lim _{t \rightarrow \infty}\left\langle\left(V(t) f-U_{0}(t) \tilde{f}_{+}\right), h\right\rangle_{\mathcal{H}}=0 .
$$

Since (A1) implies

$$
\lim _{t \rightarrow \infty}\left\langle U_{0}(t) \tilde{f}_{+}, h\right\rangle_{\mathcal{H}}=0
$$

we have

$$
\lim _{t \rightarrow \infty}\langle V(t) f, h\rangle_{\mathcal{H}}=0
$$

Hence the proof is complete.

Using Lemma 2.2 we prove Theorem as follows.

Proof of Theorem 1. We give the proof of (1) by a contradiction. Assume that there exists $f \in D(A), \lambda \in \mathbf{R}$ such that $A f=\lambda f$. Then we have

$$
\langle V(t) f, f\rangle_{\mathcal{H}}=e^{-i t \lambda}\|f\|_{\mathcal{H}}^{2} .
$$


This yields a contradiction with Lemma 2.2.

Below we prove Theorem 1 (2). First we show the existence of $W$. Set $F(\lambda)=(\lambda-i)^{-2} \lambda$. Theorem (1) and Lemma 1.1.6 of Petkov [21] or Simon [25] imply that

$$
\{F(A) f \in \mathcal{H}: f \in D(A)\} \quad \text { is dense in } \mathcal{H} .
$$

By (2.6) it is sufficient to show that $\left\{U_{0}(-t) V(t) F(A) f\right\}_{t \geqq 0}$ is Cauchy in $\mathcal{H}$ as $t \rightarrow \infty$, where $f \in D(A)$. We estimate as follows (cf. [25]):

$$
\left\|\left(U_{0}(-t) V(t)-U_{0}(-s) V(s)\right) F(A) f\right\|_{\mathcal{H}} \leqq \sum_{j=1}^{4}\left\|T_{j}\right\|_{\mathcal{H}},
$$

where

$$
\begin{aligned}
& T_{1}=U_{0}(-t)\left(F(A)-F\left(A_{0}\right)\right) V(t) f \\
& T_{2}=U_{0}(-s)\left(F(A)-F\left(A_{0}\right)\right) V(s) f \\
& T_{3}=F\left(A_{0}\right)\left(I_{d}-E_{1 / M, M}\left(A_{0}\right)\right)\left(U_{0}(-t) V(t)-U_{0}(-s) V(s)\right) f \\
& \text { and } \\
& T_{4}=F\left(A_{0}\right) E_{1 / M, M}\left(A_{0}\right)\left(U_{0}(-t) V(t)-U_{0}(-s) V(s)\right) f .
\end{aligned}
$$

We note that for any $\varepsilon$, there exists $M>1$ such that

$$
\left\|\left(1-\chi_{(-M,-1 / M) \cup(1 / M, M)}\right) F\right\|_{L^{\infty}(\mathbf{R})}<\varepsilon .
$$

Thus we have

$$
\left\|T_{3}\right\|_{\mathcal{H}}<\varepsilon\|f\|_{\mathcal{H}}
$$

By (A2), $F(A)-F\left(A_{0}\right)$ is a compact operator. Hence Lemma 2.2 implies

$$
\lim _{t \rightarrow \infty}\left\|T_{1}\right\|_{\mathcal{H}}=\lim _{s \rightarrow \infty}\left\|T_{2}\right\|_{\mathcal{H}}=0 .
$$

Lemma 2.1 implies

$$
\lim _{t, s \rightarrow \infty}\left\|T_{4}\right\|_{\mathcal{H}}=0 .
$$

(2.7), (2.8) and (2.9) imply the existence of $W$.

Next we prove $W \not \equiv 0$ (cf. [17], Section 3). Assume that $W \equiv 0$ i.e. for any $f \in \mathcal{H}$

$$
\lim _{t \rightarrow \infty}\|V(t) f\|_{\mathcal{H}}=0 .
$$


We set $G(\lambda)=(\lambda-i)^{-1}$. Then noting

$$
\begin{aligned}
& \left\langle U_{0}(-t) V(t) G(A) f, G\left(A_{0}\right) f\right\rangle_{\mathcal{H}} \\
= & \left\langle G(A) f, G\left(A_{0}\right) f\right\rangle_{\mathcal{H}}-\int_{0}^{t}\left\langle U_{0}(-\tau) B V(\tau) G(A) f, G\left(A_{0}\right) f\right\rangle_{\mathcal{H}} d \tau,
\end{aligned}
$$

we have by (2.10) and Schwarz inequality

$$
\begin{aligned}
& \left|\left\langle G(A) f, G\left(A_{0}\right) f\right\rangle_{\mathcal{H}}\right| \\
& \quad \leqq\left(\int_{0}^{\infty}\|\sqrt{B} V(\tau) G(A) f\|_{\mathcal{H}}^{2} d \tau\right)^{\frac{1}{2}} \times\left(\int_{0}^{\infty}\left\|\sqrt{B} U_{0}(\tau) G\left(A_{0}\right) f\right\|_{\mathcal{H}}^{2} d \tau\right)^{\frac{1}{2}} .
\end{aligned}
$$

(2.2) and (2.10) imply

$$
2 \int_{0}^{\infty}\|\sqrt{B} V(\tau) G(A) f\|_{\mathcal{H}}^{2} d \tau=\|G(A) f\|_{\mathcal{H}}^{2} .
$$

Hence we have by (2.11) and (2.12)

$$
\begin{aligned}
\left\|G\left(A_{0}\right) f\right\|_{\mathcal{H}}^{2} \leqq & \|f\|_{\mathcal{H}}\left\{\left\|\left(G(A)-G\left(A_{0}\right)\right) f\right\|_{\mathcal{H}}\right. \\
& \left.+\left(\frac{1}{2} \int_{0}^{\infty}\left\|\sqrt{B} U_{0}(\tau) G\left(A_{0}\right) f\right\|_{\mathcal{H}}^{2} d \tau\right)^{\frac{1}{2}}\right\} .
\end{aligned}
$$

Let fix $M>1$. Put $f=U_{0}(s) g, g$ satisfying $E_{1 / M, M}\left(A_{0}\right) g=g$. Then we have

$$
\begin{aligned}
\left\|G\left(A_{0}\right) g\right\|_{\mathcal{H}}^{2} \leqq & \|g\|_{\mathcal{H}}\left\{\left\|\left(G(A)-G\left(A_{0}\right)\right) U_{0}(s) g\right\|_{\mathcal{H}}\right. \\
& \left.+\left(\frac{1}{2} \int_{s}^{\infty}\left\|\sqrt{B} E_{1 / M, M}\left(A_{0}\right) U_{0}(\tau) G\left(A_{0}\right) g\right\|_{\mathcal{H}}^{2} d \tau\right)^{\frac{1}{2}}\right\} .
\end{aligned}
$$

(A1) and (A2) imply

$$
\lim _{s \rightarrow \infty}\left\|\left(G(A)-G\left(A_{0}\right)\right) U_{0}(s) g\right\|_{\mathcal{H}}=0 .
$$

(2.1) implies

$$
\lim _{s \rightarrow \infty} \int_{s}^{\infty}\left\|\sqrt{B} E_{1 / M, M}\left(A_{0}\right) U_{0}(\tau) G\left(A_{0}\right) g\right\|_{\mathcal{H}}^{2} d \tau=0 .
$$

Therefore it follows from $(2.13),(2.14)$ and $(2.15)$ that $g \equiv 0$. This is a contradiction. Therefore we have $W \not \equiv 0$. 
Proof of Corollary 2. First we consider the case $k=0$. Since $U_{0}(t)$ is unitary in $\mathcal{H}$, we have by Theorem 1 (2) that there exist non-trivial initial data $f \in \mathcal{H}$ such that $W f \neq 0$ and

$$
\lim _{t \rightarrow \infty}\left\|V(t) f-U_{0}(t) W f\right\|_{\mathcal{H}}=0 .
$$

Setting $f_{+}=W f$ we have Corollary 2 for $k=0$. Next we consider $k=1$. Noting the following

$$
\begin{aligned}
& \left\|V(t)\left(A-\zeta_{0}\right)^{-1} f-U_{0}(t)\left(A_{0}-\zeta_{0}\right)^{-1} f_{+}\right\|_{\mathcal{H}} \\
& \quad \leqq\left\|\left(\left(A-\zeta_{0}\right)^{-1}-\left(A_{0}-\zeta_{0}\right)^{-1}\right) V(t) f\right\|_{\mathcal{H}}+\left\|\left(A_{0}-\zeta_{0}\right)^{-1}\left(V(t) f-U_{0}(t) f_{+}\right)\right\|_{\mathcal{H}} .
\end{aligned}
$$

We have Corollary 2 for $k=1$ by Lemma 2.2, (A2) and Corollary 2 for $k=0$.

To show the cases $k=2,3,4, \cdots$, we should use the induction. Here we omit to prove.

\section{§3. Applications}

Application 1 (Acoustic wave equations with dissipative terms in stratified media).

We apply Theorem 1 (Corollary 2) to (1.1) and (1.2). We set $f(t)={ }^{t}$ $\left(u(t), \partial_{t} u(t)\right)$. Then (1.3) and (1.1) or (1.2) can be written as $\partial_{t} f=-i A_{0} f$ and $\partial_{t} f=-i A f$ respectively, where

$$
A_{0}=i\left(\begin{array}{cc}
0 & 1 \\
-L_{0} & 0
\end{array}\right), \quad A=A_{0}-i B=i\left(\begin{array}{cc}
0 & 1 \\
-L_{0} & -b(x, y)
\end{array}\right),
$$

where

$$
B=\left(\begin{array}{cc}
0 & 0 \\
0 & b(x, y)
\end{array}\right) \quad \text { or } \quad\left(\begin{array}{cc}
0 & 0 \\
0 & \langle\cdot, \varphi\rangle_{\mathcal{G}} \varphi
\end{array}\right) .
$$

Let $\mathcal{H}$ be Hilbert spaces with inner product

$$
\langle f, g\rangle_{\mathcal{H}}=\int_{\mathbf{R}^{n+1}}\left(\nabla f_{1}(x, y) \overline{\nabla g_{1}(x, y)}+f_{2}(x, y) \overline{g_{2}(x, y)} c_{0}^{-2}(y)\right) d x d y,
$$

and $\|\cdot\|_{\mathcal{H}}$ is the corresponding norm, where $f={ }^{t}\left(f_{1}, f_{2}\right), g={ }^{t}\left(g_{1}, g_{2}\right)$ and $\nabla=\left(\partial / \partial x_{1}, \partial / \partial x_{2}, \ldots, \partial / \partial x_{n}, \partial / \partial y\right)$.

The domain of $A_{0}$ is

$$
D\left(A_{0}\right)=\left\{f \in \mathcal{H} ; \triangle f_{1} \in L^{2}\left(\mathbf{R}^{n+1}\right), f_{2} \in H^{1}\left(\mathbf{R}^{n+1}\right)\right\} .
$$

Then $A_{0}$ is a self-adjoint operator in $\mathcal{H}$ and generates a unitary group $\left\{U_{0}(t)\right\}_{t \in \mathbf{R}}$ in $\mathcal{H} . B$ is non-negative and bounded.

Concerning (1.1) and (1.2) we have the following result. 
Theorem 3.1. $\quad A_{0}$ and $B$ as in (3.1) satisfy $(\mathrm{A} 1) \sim(\mathrm{A} 3)$. Therefore $A=$ $A_{0}-i B$ generates a contraction semi-group $V(t)$ and the same conclusions as in Theorem 1 and Corollary 2 hold for $V(t)$ and $U_{0}(t)$.

Proof. We make a check on (A1), (A2) and (A3). Note that

$$
T_{0} A_{0} T_{0}^{-1}=\left(\begin{array}{cc}
\sqrt{L_{0}} & 0 \\
0 & -\sqrt{L_{0}}
\end{array}\right)
$$

where

$$
T_{0}=\frac{1}{\sqrt{2}}\left(\begin{array}{cc}
\sqrt{L_{0}} & i \\
\sqrt{L_{0}} & -i
\end{array}\right)
$$

and $T_{0}$ is a unitary operator from $\mathcal{H}$ onto $\mathcal{G} \times \mathcal{G}$. It follows from $(1.5)$ that for any $u \in \mathcal{G}$

$$
\sup _{\alpha \leqq|\operatorname{Re} \zeta| \leqq \beta, 0<|\operatorname{Im} \zeta|<\eta}\left|\operatorname{Im}\left\langle\left( \pm \sqrt{L_{0}}-\zeta\right)^{-1} X_{\frac{\theta}{2}} u, X_{\frac{\theta}{2}} u\right\rangle_{\mathcal{G}}\right|<\infty .
$$

Therefore we have (A1) by Reed and Simon [24] Theorem XIII-20. By Rellich's theorem,

$$
B=\left(\begin{array}{cc}
0 & 0 \\
0 & b(x, y)
\end{array}\right) \quad \text { and } \quad\left(\begin{array}{cc}
0 & 0 \\
0 & \langle\cdot, \varphi\rangle_{\mathcal{G}} \varphi
\end{array}\right)
$$

are $A_{0}$-compact and compact operators, respectively. Therefore, since (A2) holds, we have by Reed and Simon [23] Theorem X-50 that $A$ generates a contraction semi-group $V(t)=e^{-i t A}$.

In the same argument as in Mochizuki [17] Section 3 we can show (A3) as follows. Let $g=\left(g_{1}, g_{2}\right) \in \mathcal{H}$. We set

$$
u=\left(\begin{array}{l}
u_{1} \\
u_{2}
\end{array}\right)=\left(A_{0}-\zeta\right)^{-1} \sqrt{B}\left(\begin{array}{l}
g_{1} \\
g_{2}
\end{array}\right) .
$$

First considering (1.1) i.e.

$$
B=\left(\begin{array}{cc}
0 & 0 \\
0 & b(x, y)
\end{array}\right)
$$

we have

$$
\left(L_{0}-\zeta^{2}\right) u_{2}=\zeta \sqrt{b(x, y)} g_{2}
$$

and

$$
\sqrt{B}\left(A_{0}-\zeta\right)^{-1} \sqrt{B} g=\sqrt{B} u={ }^{t}\left(0, \sqrt{b(x, y)} u_{2}\right) .
$$


Therefore we can calculate as follows:

$$
\left\|\sqrt{B}\left(A_{0}-\zeta\right)^{-1} \sqrt{B} g\right\|_{\mathcal{H}}=|\zeta|\left\|\sqrt{b(x, y)}\left(L_{0}-\zeta^{2}\right)^{-1} \sqrt{b(x, y)} g_{2}\right\|_{\mathcal{G}} .
$$

(1.5) of Proposition 1.1 and (3.3) imply (A3). Thus we have the conclusion of Theorem 1 and Corollary 2 for the between $V(t)$ by $(1.1)$ and $U_{0}(t)$.

Next we consider (1.2) i.e.

$$
B=\left(\begin{array}{cc}
0 & 0 \\
0 & \langle\cdot, \varphi\rangle_{\mathcal{G}} \varphi
\end{array}\right)
$$

Note that

$$
|\operatorname{Im} \zeta|\left\|\sqrt{B}\left(A_{0}-\zeta\right)^{-1} f\right\|_{\mathcal{H}}^{2} \leqq|\operatorname{Im} \zeta|\left\|X_{\frac{\theta}{2}}\left(\left(A_{0}-\zeta\right)^{-1} f\right)_{2}\right\|_{\mathcal{G}}^{2} \times\left\|X_{-\frac{\theta}{2}} \varphi\right\|_{\mathcal{G}}^{2}
$$

for any $f \in \mathcal{H}$.

Setting

$$
B_{1}=\left(\begin{array}{cc}
0 & 0 \\
0 & X_{\theta}
\end{array}\right)
$$

we have

$$
\begin{aligned}
|\operatorname{Im} \zeta|\left\|X_{\frac{\theta}{2}}\left(\left(A_{0}-\zeta\right)^{-1} f\right)_{2}\right\|_{\mathcal{G}}^{2} & =|\operatorname{Im} \zeta|\left\|\sqrt{B_{1}}\left(A_{0}-\zeta\right)^{-1} f\right\|_{\mathcal{H}}^{2} \\
& \leqq\left\|\sqrt{B_{1}}\left\{\left(A_{0}-\zeta\right)^{-1}-\left(A_{0}-\bar{\zeta}\right)^{-1}\right\} \sqrt{B_{1}}\right\|\|f\|_{\mathcal{H}}^{2} .
\end{aligned}
$$

Note (3.3) which is changed $B$ and $b(x, y)$ to $B_{1}$ and $X_{\theta}$, respectively. Thus (3.4) implies (1.7). Since (1.7) means that (2.1) holds, we have the conclusion of Theorem 1 (Corollary 2) for the between $V(t)$ by $(1.2)$ and $U_{0}(t)$.

Application 2 (Schrödinger equations with absorption).

Let $x \in \mathbf{R}^{n}$, where $n \geqq 1$. We assume that $V_{0}(x)$ and $\varphi_{0}(x)$ is measurable functions which satisfy

$$
0 \leqq V_{0}(x) \leqq C\left(1+|x|^{2}\right)^{-\frac{\theta}{2}}
$$

and

$$
\varphi_{0}(x) \in L^{2}\left(\mathbf{R}^{n} ;\left(1+|x|^{2}\right)^{\frac{\theta}{2}} d x\right)
$$

for some $\theta>1$ and $C>0$, respectively.

We set $\mathcal{H}=L^{2}\left(\mathbf{R}^{n}\right)$. Define

$$
\left\{\begin{array}{l}
A_{0}=-\triangle_{x}, \quad D\left(A_{0}\right)=H^{2}\left(\mathbf{R}^{n}\right), \\
\text { and } \\
B u=V_{0}(x) u(x) \quad \text { or } \quad\left\langle u, \varphi_{0}\right\rangle_{\mathcal{H}} \varphi_{0}(x)
\end{array}\right.
$$


where

$$
\triangle_{x}=\sum_{j=1}^{n} \frac{\partial^{2}}{\partial x_{j}^{2}} .
$$

$A_{0}$ is a self-adjoint operator in $\mathcal{H} . \quad B$ is non-negative and bounded operator. Thus we have $D\left(A_{0}\right) \subset D(B)$.

We consider

$$
i \partial_{t} u(x, t)=-\triangle_{x} u(x, t)-i V_{0}(x) u(x, t)
$$

and

$$
i \partial_{t} u(x, t)=-\triangle_{x} u(x, t)-i\left\langle u, \varphi_{0}\right\rangle_{\mathcal{H}} \varphi_{0}(x),
$$

where $(x, t) \in \mathbf{R}^{n} \times \mathbf{R}_{+}$.

These are similar to (1.1) and (1.2), respectively. Defining $A=A_{0}-$ $i B, D(A)=D\left(A_{0}\right)$ we write $(3.6)$ and (3.7) as follows:

$$
i \partial_{t} u(t)=A u(t)
$$

Then we have

Theorem 3.2. $\quad A_{0}$ and $B$ as in (3.5) satisfy (A1) (A3). Therefore $A=$ $A_{0}-i B$ generates a contraction semi-group $V(t)$ and the same conclusions as in Theorem 1 and Corollary 2 hold for $V(t)$ and the unitary group $U_{0}(t)$ generated by $A_{0}$.

Proof. For $A_{0}$ as in (3.5), (A1) is the well-known results. Rellich's theorem implies that operators $B$ as in (3.5) are $A_{0}$-compact and compact operators, respectively.

Finally we can show (A3). Indeed, for $B u=V_{0}(x) u(x),(1.6)$ is an immediate result from Kuroda [16] Theorem 4.4.1(2) and Corollaries 4.4.3 and 4.4.5. For $B u=\left\langle u, \varphi_{0}\right\rangle_{\mathcal{H}} \varphi_{0}(x)$, noting a similar relation as in (3.4) and [16] Theorem 4.4.1 we have (1.7). Hence the proof is complete.

Remark 3.3. We can also deal with $V_{0}(x)$ having some singularities, for instance

$$
V_{0}(x)=O\left(|x|^{-1}\right) \quad(|x| \rightarrow 0) .
$$

Then moreover assuming $n \geqq 3$ and using the Sobolev theorem

$$
H^{l}\left(\mathbf{R}^{n}\right) \hookrightarrow L^{q}\left(\mathbf{R}^{n}\right)
$$

where $1 / 2-l / n=1 / q, q>2$, we have (A2) and (A3). 
Remark 3.4. If we apply the framework as in [17] to (3.6) or (3.7) we should restrict ourselves to the cases $n \geqq 3$ and $\theta \geqq 2$ (see Proposition 3.5 as below). Because for other cases, $n=1,2$ or $1<\theta<2$, it is difficult to be held (1.8) with $A_{0}=-\triangle, B=\left(1+|x|^{2}\right)^{-\theta / 2}$ (for detail, see [16] Theorem 4.4.1 (4) and Corollaries 4.4.3, 4.4.5 and those proof). For the cases $n \geqq 3$ and $\theta \geqq 2$ we note the following proposition which is concerning the low energy part (for the high energy part, see Proposition 1.0).

Proposition 3.5. Assume that $n \geqq 3$ and $\theta \geqq 2$. Then one has

$$
\left\|Y_{\frac{\theta}{2}}\left(-\triangle_{x}-\zeta\right)^{-1} Y_{\frac{\theta}{2}}\right\|=O(1) \quad(|\zeta| \rightarrow 0),
$$

where $\zeta \in \mathbf{C}, Y_{\gamma}=\left(1+|x|^{2}\right)^{-(\gamma / 2)}$ and $\|\cdot\|$ is the norm of the bounded operator in $L^{2}\left(\mathbf{R}^{n}\right)$.

This is the well-known estimates and an improvement of [16] Theorem 4.4.1 (4) and Corollary 4.4.3 for $n \geqq 3$ and $\theta \geqq 2$. For the readers, in Appendix we give the proof which is due to that of [16] Theorem 4.4.1 (4). The conclusion in [16] Theorem 4.4.1 (4) and Corollary 4.4.3 for $n \geqq 3$ and $\theta=2$ is

$$
\left\|Y_{\frac{\theta}{2}}\left(-\triangle_{x}-\zeta\right)^{-1} Y_{\frac{\theta}{2}}\right\|=O(|\log | \zeta||) \quad(|\zeta| \rightarrow 0)
$$

\section{§4. Energy Decay States for (1.2)}

In this section we give a simple example of (1.2) which has energy decay states.

By choosing $\varphi(x, y)$ we construct complex eigenvalues and eigenvectors for

$$
A=i\left(\begin{array}{cc}
0 & 1 \\
-L_{0} & -\langle\cdot, \varphi\rangle_{\mathcal{G}} \varphi
\end{array}\right)
$$

Note that

$$
A\left(\begin{array}{l}
u \\
v
\end{array}\right)=z\left(\begin{array}{l}
u \\
v
\end{array}\right) \Longleftrightarrow\left\{\begin{array}{l}
i v=z u \\
-i L_{0} u-i\langle v, \varphi\rangle_{\mathcal{G}} \varphi=z v .
\end{array}\right.
$$

Assuming $z \neq 0$, we have

$$
L_{0} v-i z\langle v, \varphi\rangle_{\mathcal{G}} \varphi-z^{2} v=0 .
$$

Finding $z$ and $v$ which satisfy

$$
i z\langle v, \varphi\rangle_{\mathcal{G}}=1
$$


and setting

$$
\varphi=\left(L_{0}-z^{2}\right) v,
$$

we get $v$ which is a solution of (4.1). Indeed, choosing $v$ from $C_{0}^{\infty}\left(\mathbf{R}^{n+1}\right)$ and introducing (4.3) to (4.2) we have

$$
\|\nabla v\|_{L^{2}\left(\mathbf{R}^{n+1}\right)}^{2}-\bar{z}^{2}\|v\|_{\mathcal{G}}^{2}=\frac{1}{i z}\left(=\frac{-i \bar{z}}{|z|^{2}}\right) .
$$

Put $z=\lambda+i \kappa, \lambda \in \mathbf{R}, \kappa<0$. Then it follows from the real and imaginary parts of (4.4) that

$$
\|\nabla v\|_{L^{2}\left(\mathbf{R}^{n+1}\right)}^{2}-\left(\lambda^{2}-\kappa^{2}\right)\|v\|_{\mathcal{G}}^{2}=\frac{-\kappa}{\lambda^{2}+\kappa^{2}}
$$

and

$$
2 \lambda \kappa\|v\|_{\mathcal{G}}^{2}=\frac{-\lambda}{\lambda^{2}+\kappa^{2}} \Longleftrightarrow \lambda=0 \quad \text { or } \quad 2 \kappa\|v\|_{\mathcal{G}}^{2}=\frac{-1}{\lambda^{2}+\kappa^{2}} .
$$

Thus we have by (4.5) and (4.6) that

$$
\left\{\begin{array}{l}
\lambda=0 \\
\kappa^{3}\|v\|_{\mathcal{G}}^{2}+\kappa\|\nabla v\|_{L^{2}\left(\mathbf{R}^{n+1}\right)}^{2}+1=0
\end{array}\right.
$$

or

$$
\left\{\begin{array}{c}
\|\nabla v\|_{L^{2}\left(\mathbf{R}^{n+1}\right)}^{2}-\left(\lambda^{2}-\kappa^{2}\right)\|v\|_{\mathcal{G}}^{2}=2 \kappa^{2}\|v\|_{\mathcal{G}}^{2} \\
\left(\Longleftrightarrow \frac{1}{\lambda^{2}+\kappa^{2}}=\frac{\|v\|_{\mathcal{G}}^{2}}{\|\nabla v\|_{L^{2}\left(\mathbf{R}^{n+1}\right)}^{2}}\right) \\
2 \kappa\|v\|_{\mathcal{G}}^{2}=\frac{-1}{\lambda^{2}+\kappa^{2}}
\end{array}\right.
$$

The above equation implies

$$
\left\{\begin{array}{l}
\lambda= \pm \frac{\sqrt{\left(2\|\nabla v\|_{L^{2}\left(\mathbf{R}^{n+1}\right)}^{2}-1\right)\left(2\|\nabla v\|_{L^{2}\left(\mathbf{R}^{n+1}\right)}^{2}+1\right)}}{2\|\nabla v\|_{L^{2}\left(\mathbf{R}^{n+1}\right)} \times\|v\|_{\mathcal{G}}} \\
\kappa=\frac{-1}{2\|\nabla v\|_{L^{2}\left(\mathbf{R}^{n+1}\right)}} .
\end{array}\right.
$$

For any non-trivial $v \in C_{0}^{\infty}\left(\mathbf{R}^{n+1}\right)$, the equation of $\kappa$ in (4.7) has a unique solution $\kappa<0$. Therefore we have

$$
A\left(\begin{array}{c}
\frac{1}{\kappa} v \\
v
\end{array}\right)=i \kappa\left(\begin{array}{c}
\frac{1}{\kappa} v \\
v
\end{array}\right)
$$

and

$$
V(t)\left(\begin{array}{c}
\frac{1}{\kappa} v \\
v
\end{array}\right)=e^{\kappa t}\left(\begin{array}{c}
\frac{1}{\kappa} v \\
v
\end{array}\right)
$$


Moreover assuming that $u$ satisfies $\|\nabla v\|_{L^{2}\left(\mathbf{R}^{n+1}\right)}>1 / \sqrt{2}$, we also have by (4.8)

$$
A\left(\begin{array}{c}
\frac{1}{\lambda+i \kappa} v \\
v
\end{array}\right)=(\lambda+i \kappa)\left(\begin{array}{c}
\frac{1}{\lambda+i \kappa} v \\
v
\end{array}\right)
$$

and

$$
V(t)\left(\begin{array}{c}
\frac{1}{\lambda+i \kappa} v \\
v
\end{array}\right)=e^{(-i \lambda+\kappa) t}\left(\begin{array}{c}
\frac{1}{\lambda+i \kappa} v \\
v
\end{array}\right),
$$

where $\lambda$ and $\kappa$ are as in (4.8).

In the same way as above we also have an energy decay state of

$$
i \partial_{t} u(x, t)=-\triangle_{x} u(x, t)-(\alpha+i \beta)\left\langle u, \varphi_{0}\right\rangle_{L^{2}\left(\mathbf{R}^{n}\right)} \varphi_{0}(x),
$$

where $(x, t) \in \mathbf{R}^{n} \times \mathbf{R}_{+}, \alpha \in \mathbf{R}, \beta>0$ and $\varphi_{0}(x)$ is same as in Application 2 .

Indeed, setting

$$
\begin{aligned}
\lambda & =\frac{\left\|\nabla u_{0}\right\|_{L^{2}\left(\mathbf{R}^{n}\right)}^{2}}{\left\|u_{0}\right\|_{L^{2}\left(\mathbf{R}^{n}\right)}^{2}}-\frac{\alpha}{\alpha^{2}+\beta^{2}} \times \frac{1}{\left\|u_{0}\right\|_{L^{2}\left(\mathbf{R}^{n}\right)}^{2}} \\
\kappa & =\frac{-\beta}{\alpha^{2}+\beta^{2}} \times \frac{1}{\left\|u_{0}\right\|_{L^{2}\left(\mathbf{R}^{n}\right)}^{2}}
\end{aligned}
$$

and

$$
\varphi_{0}=\left(-\triangle_{x}-\lambda-i \kappa\right) u_{0}
$$

for non-trivial $u_{0} \in C_{0}^{\infty}\left(\mathbf{R}^{n}\right)$, we have

$$
-\triangle_{x} u_{0}-(\alpha+i \beta)\left\langle u_{0}, \varphi_{0}\right\rangle_{L^{2}\left(\mathbf{R}^{n}\right)} \varphi_{0}=(\lambda+i \kappa) u_{0} .
$$

Therefore we have an energy decay state of (4.11).

\section{Appendix}

Proof of Proposition 3.5. Note that $|\zeta|$ is small. We consider the following decomposition:

$$
\begin{aligned}
& Y_{\frac{\theta}{2}}\left(-\triangle_{x}-\zeta\right)^{-1} Y_{\frac{\theta}{2}} \\
& \quad=\left(\int_{0}^{\frac{|\zeta|}{2}}+\int_{\frac{|\zeta|}{2}}^{\frac{3|\zeta|}{2}}\right) \frac{\mu^{-\frac{1}{2}}}{\mu-\zeta} Y_{\frac{\theta}{2}} t\left(\mu^{\frac{1}{2}}\right)^{*} t\left(\mu^{\frac{1}{2}}\right) Y_{\frac{\theta}{2}} d \mu+Y_{\frac{\theta}{2}}\left(-\triangle_{x}-\zeta\right)^{-1} E\left(\left(\frac{3|\zeta|}{2}, \infty\right)\right) Y_{\frac{\theta}{2}},
\end{aligned}
$$

where $t\left(\mu^{1 / 2}\right)$ is a trace operator, from $L^{2}\left(\mathbf{R}^{n} ;\left(1+|x|^{2}\right)^{-(\theta / 2)} d x\right)$ to $L^{2}\left(\mathbf{S}^{n-1}\right)$, given by

$$
t\left(\mu^{\frac{1}{2}}\right) v(\omega)=2^{-\frac{1}{2}} \mu^{\frac{n-1}{4}}(2 \pi)^{-\frac{n}{2}} \int_{\mathbf{R}^{n}} e^{-i x \cdot \mu^{\frac{1}{2}} \omega} v(x) d x
$$


for $v \in C_{0}^{\infty}\left(\mathbf{R}^{n}\right)$ and $E(\lambda)$ is the spectral family of $A_{0}=-\triangle_{x}$.

[16] has already gotten

$$
\left\|\int_{0}^{\frac{|\zeta|}{2}} \frac{\mu^{-\frac{1}{2}}}{\mu-\zeta} Y_{\frac{\theta}{2}} t\left(\mu^{\frac{1}{2}}\right)^{*} t\left(\mu^{\frac{1}{2}}\right) Y_{\frac{\theta}{2}} d \mu\right\|=O(1)
$$

and

$$
\left\|\int_{\frac{|\zeta|}{2}}^{\frac{3|\zeta|}{2}} \frac{\mu^{-\frac{1}{2}}}{\mu-\zeta} Y_{\frac{\theta}{2}} t\left(\mu^{\frac{1}{2}}\right)^{*} t\left(\mu^{\frac{1}{2}}\right) Y_{\frac{\theta}{2}} d \mu\right\|=O(1)
$$

as $|\zeta| \rightarrow 0$ (see the proof of Theorem 4.4 .1 of [16]).

Next, note that the well-known inequality

$$
\int_{\mathbf{R}^{n}} \frac{|u(x)|^{2}}{|x|^{2}} d x \leqq C\left|\left\|\nabla_{x} \mid u\right\|_{L^{2}\left(\mathbf{R}^{n}\right)}^{2}\right.
$$

for some $C>0$.

Then we can calculate as follows. For any $u \in \mathcal{H}=L^{2}\left(\mathbf{R}^{n}\right)$ we have

$$
\begin{aligned}
& \left\|Y_{\frac{\theta}{2}}\left(-\triangle_{x}-\zeta\right)^{-1} E\left(\left(\frac{3|\zeta|}{2}, \infty\right)\right) Y_{\frac{\theta}{2}} u\right\|_{\mathcal{H}} \\
& \quad \leqq C\left\|\left|\nabla_{x}\right|\left(-\triangle_{x}-\zeta\right)^{-1} E\left(\left(\frac{3|\zeta|}{2}, \infty\right)\right) Y_{\frac{\theta}{2}} u\right\|_{\mathcal{H}}
\end{aligned}
$$

and

$$
\begin{aligned}
& \left\|Y_{\frac{\theta}{2}}\left|\nabla_{x}\right|\left(-\triangle_{x}-\bar{\zeta}\right)^{-1} E\left(\left(\frac{3|\zeta|}{2}, \infty\right)\right) u\right\|_{\mathcal{H}} \\
& \quad \leqq C\left\|-\triangle_{x}\left(-\triangle_{x}-\bar{\zeta}\right)^{-1} E\left(\left(\frac{3|\zeta|}{2}, \infty\right)\right) u\right\|_{\mathcal{H}} .
\end{aligned}
$$

Since

$$
\left(\left|\nabla_{x}\right|\left(-\triangle_{x}-\zeta\right)^{-1} E\left(\left(\frac{3|\zeta|}{2}, \infty\right)\right) Y_{\frac{\theta}{2}}\right)^{*}=Y_{\frac{\theta}{2}}\left|\nabla_{x}\right|\left(-\triangle_{x}-\bar{\zeta}\right)^{-1} E\left(\left(\frac{3|\zeta|}{2}, \infty\right)\right),
$$

we have

$$
\begin{aligned}
\left\|Y_{\frac{\theta}{2}}\left(-\triangle_{x}-\zeta\right)^{-1} E\left(\left(\frac{3|\zeta|}{2}, \infty\right)\right) Y_{\frac{\theta}{2}}\right\| & \leqq C\left\|-\triangle_{x}\left(-\triangle_{x}-\bar{\zeta}\right)^{-1} E\left(\left(\frac{3|\zeta|}{2}, \infty\right)\right)\right\| \\
& =\left\|\left(I_{d}+\bar{\zeta}\left(-\triangle_{x}-\bar{\zeta}\right)^{-1}\right) E\left(\left(\frac{3|\zeta|}{2}, \infty\right)\right)\right\| \\
& =O(1) \quad(|\zeta| \rightarrow 0) .
\end{aligned}
$$

Now the proof is complete. 


\section{References}

[1] Ben-Artzi, M., Dermenjian, Y. and Guillot, J. C., Acoustic waves in perturbed stratified fluids: a spectral theory, Comm. Partial Diff. Equations, 14 (1989), 479-517.

[2] - Analyticity properties and estimates of resolvent kernels near thresholds, Comm. Partial Diff. Equations, 25 (2000), 1753-1770.

[3] Boutet de Monvel-Berthier, A. and Manda, D., Spectral and scattering theory for wave propagation in perturbed stratified media, J. Math. Anal. Appl., 191 (1995), 137-167.

[4] Dermenjian, Y. and Guillot, J. C., Scattering of elastic waves in a perturbed isotropic half space with a free boundary. The limiting absorption principle, Math. Meth. Appl. Sci., 10 (1988), 87-124.

[5] DeBièvre, S. and Pravica, W., Spectral analysis for optical fibers and stratified fluids I: The limiting absorption principle, J. Funct. Anal., 98 (1991), 404-436.

[6] - Spectral analysis for optical fibers and stratified fluids II: Absence of eigenvalues, Comm. Partial Diff. Equations, 17 (1992), 69-97.

[7] Eidus, D., The limiting absorption and amplitude principle for the diffraction problem with two unbouned media, Comm. Math. Phys., 107 (1986), 29-38.

[8] Enss, V., Asymptotic completeness for quantum mechanical potential scattering, Comm. Math. Phys., 61 (1978), 285-291.

[9] Georgiev, V., Disappearing solutions for dissipative hyperbolic systems of constant multiplicity, Hokkaido Math. J., 15 (1986), 357-385.

[10] Kadowaki, M., The limiting absorption principle for the acoustic wave operators in two unbounded media, Tsukuba. J. Math., 17 (1993), 345-362.

[11] - Asymptotic completeness for acoustic propagators in perturbed stratified media, Integral Equations Operator Theory, 26 (1996), 432-459.

[12] Low and high energy resolvent estimates for wave propagation in stratified media and their applications, to appear in J. Diff. Equations.

[13] — On a framework of scattering for dissipative systems, Preprint.

[14] Kato, T., Wave operator and similarity for some non-selfadjoint operators, Math. Ann., 162 (1966), 258-279.

[15] Kato, T. and Yajima, K., Some examples of smooth operators and the associated smoothing effect, Rev. Math. Phys., 4 (1989), 481-496.

[16] Kuroda, S. T., An Introduction to Scattering Theory, Lecture Note Series $N^{o} 51$, Matematisk Institut. Aarhus University, 1980.

[17] Mochizuki, K., Scattering theory for wave equations with dissipative terms, Publ. RIMS, Kyoto Univ., 12 (1976), 383-390.

[18] Mochizuki, K. and Nakazawa, H., Energy decay and asymptotic behavior of solutions to the wave equations with linear dissipation, Publ. RIMS, Kyoto Univ., 32 (1996), 401-414.

[19] Nakazawa, H., Scattering theory for the dissipative wave equations in two space dimension, Preprint.

[20] Perry, P. A., Scattering theory by the Enss method, Hawood Academic, London, 1983.

[21] Petkov, V., Scattering theory for hyperbolic operators, North-Holland, Amsterdam, New York, Oxford, Tokyo, 1989.

[22] Shimizu, S., Scattering theory for elastic wave propagation problem in perturbed stratified media II, Publ. RIMS, Kyoto Univ., 33 (1997), 341-358.

[23] Reed, M. and Simon, B., Methods of Modern Mathematical Physics, II. Fourier Analysis, Self-Adjointness, Academic Press, New York, San Francisco, London, 1975.

[24] - Methods of Modern Mathematical Physics, IV, Analysis of Operators, Academic Press New York, San Francisco, London, 1978.

[25] Simon, B., Phase space analysis of simple scattering systems: extensions of some work of Enss, Duke Math. J., 46 (1979), 119-168.

[26] Stefanov, P. and Georgiev, V., Existence of the scattering operator for dissipative hyperbolic systems with variable multiplicities, J. Operator Theory, 19 (1988), 217-241. 
[27] Weder, R., Spectral and scattering theory for wave propagation in perturbed stratified media, Applied Mathematical Sciences 87, Springer-Verlag, New York, Berlin, Heidelberg, 1991.

[28] Wilcox, C., Sound propagation in stratified fluids, Applied Mathematical Sciences 50, Springer-Verlag, New York, Berlin, Heidelberg, 1984.

[29] Zhang, B., Commutator estimate, Besov spaces and scattering problems for the acoustic wave propagation in perturbed stratified fluids, Math. Proc. Camb. Phil. Soc., 128 (2000), 177-192. 